\title{
Effects of ethanol withdrawal on ethanol-reinforced responding in rhesus monkeys
}

\author{
Gail Winger \\ Department of Pharmacology, University of Michigan, Ann Arbor, Michigan, 48109 (U.S.A.)
}

(Received August 1st, 1988)

\begin{abstract}
Rhesus monkeys self-administered ethanol intravenously during daily, 3 - $h$ sessions. When ethanol-reinforced responding was stable and ethanol intake was in the range of $2.6-3.6 \mathrm{~g} / \mathrm{kg} / 3 \mathrm{~h}$, physiological dependence to ethanol was induced by daily passive infusions of additional ethanol. In less than 1 week, mild to moderate withdrawal signs were observed prior to daily sessions. Ethanol intake was suppressed in the presence of these withdrawal signs and returned to normal only after withdrawal signs had subsided.
\end{abstract}

Key words: ethanol-reinforced responding; physiological dependence; rhesus monkeys

\section{Introduction}

Ethanol, barbiturates, and opioids are among the centrally acting drugs that are subject to abuse; these drugs also produce pronounced withdrawal signs when their administration is discontinued in physiologically dependent individuals. The relation between drug selfadministration and drug withdrawal signs has been the focus of much theory, speculation [1] and research [e.g., $2-5$ ]. Since drug administration can relieve the discomfort of drug with drawal signs, it has been supposed that the drug gains additional reinforcing strength in the organism that is experiencing drug withdrawal signs. Whereas there is some evidence to support this idea in the case of opioids $[2,3,6]$, attempts to demonstrate an enhanced reinforcing effect of ethanol during ethanol withdrawal have usually [e.g., 4,7], although not always [5], been unsuccessful.

Both experimental and anecdotal reports of human ethanol consumption indicate that ethanol drinking by abusers may not consistently occur in the presence of ethanol withdrawal. The pattern of human ethanol consumption known as 'binge' or 'spree' drinking is typified by ingestion of large amounts of ethanol over relatively short periods of time, following which, there is a decrease in the amount of ethanol ingested and ethanol withdrawal signs may develop. This 'binge' pattern of drinking has been shown in studies of human ethanol consumption under experimentally monitored but relatively free access conditions. Periods of intoxication with the development of dependence were frequently followed by periods of reduced drinking and withdrawal signs that the subjects did not ameliorate by sustaining or increasing their ethanol consumption $[8,9]$.

Studies of intravenous ethanol intake in rhesus monkeys have produced results strikingly similar to those shown in humans $[10,11]$. Monkeys allowed free access to intravenous ethanol, self-administered large quantities of ethanol for a week or less and then ceased responding and developed mild to moderate withdrawal signs. After a day or two, the monkeys reinitiated ethanol-reinforced responding and either showed subsequent, similar episodes of termination of ethanol- 
reinforced responding, or maintained high ethanol intake levels and developed problems of ethanol toxicity. When ethanol availability was limited to $3 \mathrm{~h}$ each day, no cyclicity of intake occurred, nor were there any observable withdrawal signs. Problems of ethanol toxicity, particularly anorexia, which were profound under conditions of unlimited access, were markedly reduced under 3 -h access conditions.

These data suggest a close association between ethanol withdrawal and termination of ethanol-reinforced responding. The present experiment was designed to measure more directly the effects of ethanol deprivation on ethanol self-administration. The relation between the severity of withdrawal and the amount of ethanol self-administered was of experimental interest. Since, in monkeys under these conditions, ethanol withdrawal signs show peak severity within $24 \mathrm{~h}$ after the last dose of ethanol and gradually recover during the following several days, withdrawal severity was manipulated by varying the number of hours of ethanol deprivation in the ethanoldependent monkey. The amount of ethanol selfadministered by the monkey, given a specified length of ethanol deprivation, was the primary datum in these observations.

\section{Methods and Procedure}

Subjects were three juvenile male rhesus monkeys, surgically prepared with intravenous catheters [12]. The catheters were protected by steel harnesses and jointed restraining arms as described by Deneau et al. [12]. This equipment also served to restrain the monkeys within their individual experimental chambers, which were wooden cubicles measuring approx. $64 \times$ $70 \times 77 \mathrm{~cm}$ deep. Each chamber was equipped with a response lever and an array of stimulus lights. The lights signalled the various components of the experimental session. The intravenous catheters were attached to WatsonMarlow infusion pumps (Model MHRK 55, Falmouth, U.K.) that could be operated remotely. Control of the experiment and data collection were accomplished with a PDP8/e computer.
The daily experimental session consisted of three distinct components. During the first 15 to $25 \mathrm{~min}$ of a session, food was delivered contingently on lever-press responses. The schedule of food reinforcement was a chain fixed interval $5 \mathrm{~min}$, fixed ratio 30 . During the fixedinterval portion of the chain, both a green and a white light were illuminated in the chamber. The first response made after $5 \mathrm{~min}$ in this stimulus condition turned off the white light. In the presence of the green light alone, each 30 responses resulted in delivery of one $300 \mathrm{mg}$, banana-flavored food pellet. A total of 10 food pellets could be earned in each fixed ratio portion of this schedule. The food-availability portion of a daily session continued until 20 pellets had been earned, or $25 \mathrm{~min}$ had passed, whichever occurred first. A red light was then illuminated, signalling ethanol availability under a fixed ratio schedule. During the 3 -h period of ethanol availability, ethanol $(15 \% \mathrm{w} / \mathrm{v})$ at a dose of $0.1 \mathrm{~g} / \mathrm{kg} /$ injection was delivered i.v. following each 10 (for monkey $\mathrm{Sn}$ ) or 30 (for monkeys $\mathrm{Ro}$ and $\mathrm{He}$ ) responses. No limit except that of time was placed on the amount of ethanol that could be self-administered. The animals were fed a full ration of Purina Monkey Chow following the session and were not maintained at reduced body weight.

This schedule of responding maintained by food and ethanol was evaluated for several weeks in each monkey to allow behavior to stabilize. After ethanol-reinforced responding was occurring regularly during the 3 -h period of ethanol availability, the process of producing physiological dependence was begun. Initially, a supplemental infusion of $40 \%(w / v)$ ethanol was given $8 \mathrm{~h}$ after the end of each session. This infusion was delivered i.v. by a Sage (Model 249-2) infusion pump at a rate of approximately $10 \mathrm{ml} / \mathrm{min}$. The dose delivered was no greater than $3 \mathrm{~g} / \mathrm{kg}$ and was less if, at a lower dose, the monkey was rendered unconscious and did not blink in response to a touch near his eye.

During periods of chronic ethanol infusion, the monkeys were observed daily immediately prior to each session and following each session. Each monkey was graded on a scale of one to four as to the presence of ataxia and pres- 
ence of tremor, using a grading system that has been used and validated previously in this laboratory [13].

Within 2-4 days after the initiation of the 8$h$ post-session ethanol infusions, the monkeys completely ceased both ethanol- and food-reinforced responding during the daily sessions. Corresponding closely in time to the cessation of ethanol-reinforced responding was the appearance of mild withdrawal tremors. These tremors were most obvious following the daily session (no ethanol was being self-administered), $16 \mathrm{~h}$ after the passive administration of a high dose of ethanol. Since the monkeys were no longer self-injecting ethanol during the daily session, additional passive infusions of ethanol of as much as $3 \mathrm{~g} / \mathrm{kg}$ ethanol were given immediately after each session. Thus, the monkeys were passively given high doses of ethanol twice daily, the first dose immediately following the session and the second dose approx. $8 \mathrm{~h}$ after the first.

Following at least 7 days of chronic twicedaily ethanol administration, observations of the effects of various ethanol deprivation times on tremor scores and ethanol intake were begun. The ethanol-deprivation times were 0 (a $1 \mathrm{~g} / \mathrm{kg}$ dose of $40 \%$ ethanol was infused immediately before a session was begun), 12 (typical session conditions), $21,45,69,93$ and $117 \mathrm{~h}$ following the last administered dose of ethanol. The order of withdrawal times was unsystematic and different for each monkey. Each monkey was exposed to each deprivation time on one occasion (the 93-h period was evaluated in only two of the monkeys). Deprivation conditions were imposed by simply omitting the passive ethanol infusions and the daily session opportunities for food- and ethanol-reinforced responding for the selected number of hours. In the case of 45-h deprivation, for example, a normal session and immediate post-session infusion occurred on day 1 . The $8 \mathrm{~h}$ postsession infusion was omitted on this day; the session, the immediate post-session infusion and the 8-h post-session infusion were omitted on the following day. The monkeys were then evaluated for degree of tremor and the opportunity for ethanol-reinforced responding was made available at the normal session time on the third day, $45 \mathrm{~h}$ after the last infusion of ethanol.

One monkey (Sn), after being exposed to his first withdrawal condition (96 $\mathrm{h}$ withdrawal), began again to self-administer ethanol and food during the daily sessions, even though ethanol continued to be passively administered $8 \mathrm{~h}$ after each session. At this time, he no longer showed withdrawal tremors prior to a daily session. He did not show withdrawal tremors following $24 \mathrm{~h}$ of ethanol deprivation and selfadministered $3.9 \mathrm{~g} / \mathrm{kg}$ ethanol at this deprivation point. In order to assure consistent development of physiological dependence in this monkey, an additional passive infusion of ethanol was given to him $4 \mathrm{~h}$ before the session. After 3 days of twice-daily ethanol infusions, this monkey stopped self-administering ethanol during the session and began to show pre- and post-session withdrawal tremors. At this time, post-session ethanol infusions were instituted in this monkey as well, so that he was receiving ethanol infusions every $8 \mathrm{~h}$, a schedule that has been used previously to produce physiological dependence in monkeys [13]. Because this monkey, on a typical experimental day, was evaluated under 4 -h rather than 12 -h ethanol deprivation conditions, a 12-h deprivation test was conducted on him by eliminating the $4 \mathrm{~h}$ infusion on 1 day. The 24 -h deprivation point was redetermined in this monkey and only the data obtained on the redetermination were included in the analysis.

Between each observation of the effect of a withdrawal period (except the 0 hour deprivation condition), at least 6 days of the baseline condition of daily ethanol infusions and daily opportunities to self-administer ethanol were reinstated to insure that physiological dependence had been re-established.

\section{Results}

Prior to the initiation of passive ethanol infusions, each monkey self-administered substantial amounts of ethanol during each 3-h session. Over a 5 day period, just prior to commencing passive ethanol infusions, monkey $\mathrm{Sn}$ self- 
injected an average of $3.64 \pm 0.12 \mathrm{~g} / \mathrm{kg}$ ethanol/ $3 \mathrm{~h}$, monkey Ro self-injected an average of 3.02 $\pm 0.09 \mathrm{~g} / \mathrm{kg}$ ethanol $/ 3 \mathrm{~h}$, and monkey He selfinjected an average of $2.80 \pm 0.12 \mathrm{~g} / \mathrm{kg}$ ethanol/ $3 \mathrm{~h}$. Rates of food-reinforced responding were relatively stable within a single monkey but variable among the monkeys during this time. Rates of responding during the first fixed ratio link of the chain schedule of food-reinforced behavior averaged $0.07 \pm 0.01$ responses $/ \mathrm{s}$ for $\mathrm{Sn}$ in the pre-ethanol period and $0.09 \pm 0.03$ responses/s in the post-ethanol period. For monkey Ro, these rates were $0.46 \pm 0.17$ and $0.04 \pm 0.04$ responses $/ \mathrm{s}$, and for monkey $\mathrm{He}$, these rates were $1.07 \pm 0.11$ and $1.2 \pm 0.09$ responses/s. Because of the between-subjects variability of the response rates, the lack of food deprivation conditions, and the potential suppression of food ingestion by ethanol, the data on food-reinforced responding are not emphasized here.

Following introduction of 8 -h post session ethanol infusions, rates of both food and ethanol-reinforced responding decreased dramatically. Monkey Sn made no responses during the session, for either food or ethanol, during the first 7 days following initiation of passive ethanol infusions. Monkeys Ro and $\mathrm{He}$ decreased their session responses to nearly zero by the third day of passive ethanol infusions.

The effects on tremor scores and ethanol self-administration of withdrawing ethanol following at least 6 days of chronic passive administration of ethanol are shown in Fig. 1. Presession withdrawal scores peaked at $21 \mathrm{~h}$ of ethanol deprivation. Tremor scores were also elevated at $12 \mathrm{~h}$ of ethanol deprivation. No ethanol injections were taken by any of the monkeys following $12 \mathrm{~h}$ of withdrawal and an average of less than one injection was taken by monkeys at the 21-h deprivation point. Forty five and $69 \mathrm{~h}$ of ethanol deprivation resulted in less withdrawal tremor, as withdrawal signs were declining, and an average of 10 and 12 injections of ethanol were self-administered at these withdrawal periods, respectively. At 93 and $117 \mathrm{~h}$ of ethanol deprivation, no tremors or other signs of withdrawal were observed, and
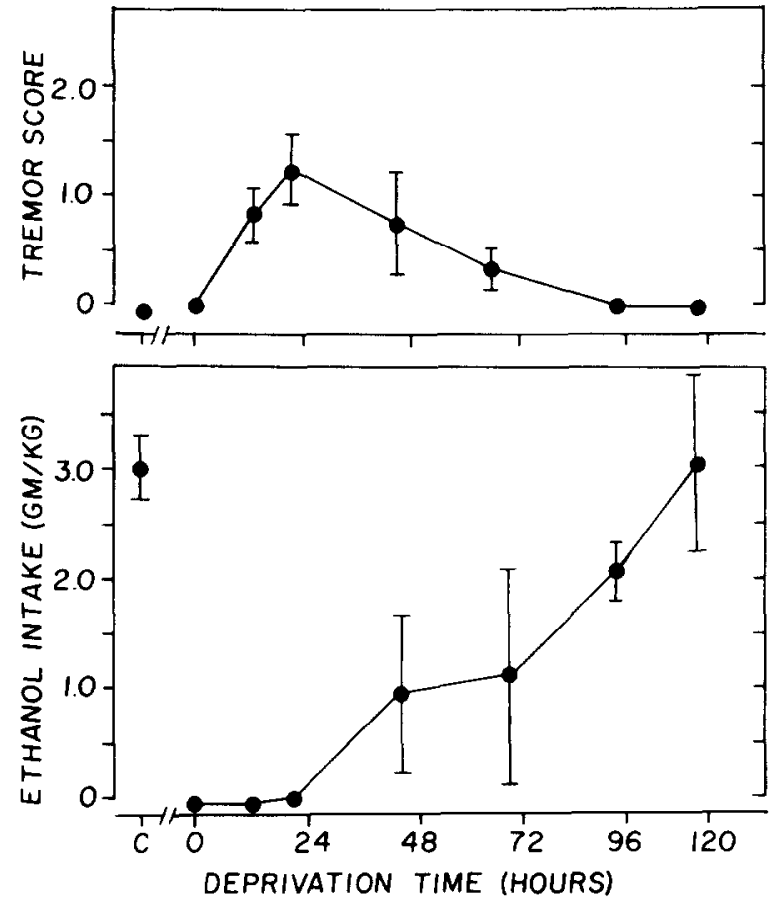

Fig. 1. The effect of ethanol deprivation in dependent monkeys on the amount of pre-session hand tremor (top) and the amount of ethanol self-administered in $\mathrm{g} / \mathrm{kg} / 3 \mathrm{~h}$ session (bottom). Each ethanol injection was $0.1 \mathrm{~g} / \mathrm{k}$. The point at $\mathrm{C}$ indicates the average ethanol intake in the 10 daily sessions immediately prior to initiation of chronic ethanol administration. All other points represent the average data from three monkeys, with a single observation in each monkey. At the 93-h deprivation time, only two monkeys were studied. The brackets are \pm 1 S.E.M.

the monkeys self-administered an average of 3.1 and $3.2 \mathrm{~g} / \mathrm{kg}$ ethanol $/ 3 \mathrm{~h}$ at these respective withdrawal times, closely approximating the average intake occurring prior to the establishment of ethanol dependence.

In the two monkeys with low rates of foodreinforced responding, the rates of food-reinforced responding were zero when ethanolreinforced responding was completely suppressed and tended to return to more normal, low rates with the longer deprivation conditions. With monkey He, rates of food-reinforced responding did not correspond to rates of ethanol-reinforced responding. He responded at a high rate during the food portion of the schedule following $45 \mathrm{~h}$ of deprivation, for example, and did not respond at all during the 
food-reinforced portion of the schedule following $69 \mathrm{~h}$ of deprivation.

\section{Discussion}

In general, these data indicate that ethanolreinforced responding decreases in relation to the severity of ethanol withdrawal. Two interesting discrepancies occurred however, one at the 0 -h deprivation condition, and another at the 45- and 69-h deprivation conditions. Under the 0 -h deprivation condition, the monkeys had received $1 \mathrm{~g} / \mathrm{kg}$ of ethanol immediately prior to the start of the session. This was sufficient ethanol to reverse all withdrawal signs in this situation, but was not enough ethanol to suppress completely ethanol-reinforced responding under similar conditions [14]. Yet, none of the monkeys in this experiment showed any ethanol self-administration at this time. Similarly, at 45 and $69 \mathrm{~h}$ of deprivation, the monkeys self-administered approx. $1 \mathrm{~g} / \mathrm{kg}$ of ethanol. This was presumably enough ethanol to reverse the withdrawal signs, but the monkeys did not then proceed to continue ethanol administration and become more profoundly intoxicated as they did at longer withdrawal times.

These discrepancies detract from a simple hypothesis suggesting that ethanol loses its' ability to maintain responding because ethanol withdrawal signs develop, and that decreases in ethanol-reinforced responding are directly related to the current degree of ethanol withdrawal. Another hypothesis that these data support more readily is that ethanol withdrawal signs and the suppression of ethanol self-administration result from a common physiological derangement, for example, buildup of a biochemical product. If the effects of such a product are triggered by ethanol deprivation, but, in contrast to other aspects of withdrawal, are not reversed by ethanol administration, they could be used to explain the current data. At the $0 \mathrm{~h}$ deprivation time, as well as at 12 and $21 \mathrm{~h}$ of deprivation, the levels of this compound would be high, reducing the reinforcing effects of ethanol. At 45 and $69 \mathrm{~h}$ of withdrawal, the levels would be slightly decreased and some ethanol-reinforced responding would occur. By $117 \mathrm{~h}$ of deprivation, this compound would have disappeared and ethanol intake would return to normal.

These data point out, whether this hypothesis holds or not, that the reinforcing efficacy of ethanol is not increased in the presence of ethanol withdrawal under conditions described in this study. If the similarities shown in previous studies between patterns of ethanol intake in rhesus monkeys and humans are a result of similar responses to ethanol withdrawal, these data suggest that ethanol may lose its' reinforcing effects in humans during ethanol withdrawal. It is possible that the cyclic patterns of ethanol intake observed in both these species may be related to onset of withdrawal. Perhaps, if sufficient ethanol deprivation occurs, due, for example, to several hours of sleep or a period of insufficient means to obtain ethanol, withdrawal signs develop and ethanol self-administration subsequently does not continue until these signs have subsided. Some investigators of experimental ethanol intake in humans have observed that termination of ethanol self-administration frequently occurs in the presence of stressful situations [15]. Ethanol withdrawal itself may be such a stressful situation.

This is not to suggest that ethanol withdrawal will always result in decreased ethanol intake in human or non-human consumers of ethanol. Important variables such as history of ethanol self-administration, and experience in reversing ethanol withdrawal with ethanol ingestion were not evaluated in this study and may be critical. Also, degree of withdrawal even milder than that described here could potentially result in increased rather than decreased levels of ethanol ingestion. These issues remain to be evaluated.

\section{Acknowledgement}

This work was supported in part by PHSADAMHA grants DA 00254, DA 04403 and AA 07201. The author is grateful to Dr. J.H. Woods for his comments during preparation of the manuscript. 


\section{References}

1 J.R. Nichols, Sci. Am., 212 (1965) 80.

2 J.H. Woods et al., Changes in operant behavior during deprivation- and antagonist-induced withdrawal states, in: L. Goldberg and F. Hoffmeister (Eds.), Psychic Dependence, Springer-Verlag, Heidelberg. 1973 , p. 114.

3 C.R. Schuster and J.H. Woods, Int. J. Addict., 223 (1968) 223.

4 M.E. Heintzelman et al., Science, 191 (1976) 482.

5 J.L. Falk and H.H. Samson, Pharmacol. Rev., 227 (1976) 449.

6 J.H. Woods and C.R. Schuster, Opiates as reinforcers, in: T. Thompson and R. Pickens (Eds.), The Stimulus Properties of Drugs, Appleton-Century-Crofts, New York, 1968, p. 163.

7 R.D. Myers et al., Physiol. Behav., 9 (1972) 43.
8 N.K. Mello and J.H. Mendelson, Psychosom. Med., 34 (1972) 139.

9 P.E. Nathan et al., Arch. Gen. Psychiatry, 22 (1970) 419.

10 G.D. Winger and J.H. Woods, Ann. N.Y. Acad. Sci., 215 (1973) 162 .

11 J.H. Woods et al., The reinforcing property of ethanol, in: M.K. Roach et al. (Eds.), Biological Aspects of Alcohol, University of Texas Press, Austin, 1971, p. 371.

12 G.A. Deneau et al., Psychopharmacologia (Berlin), 16 (1969) 30.

13 J.S. Tarika and G. Winger, Psychopharmacology (Berlin), 70 (1980) 201.

14 A.J. Karoly et al., Psychopharmacology (Berlin), 58 (1978) 19.

15 J.H. Mendelson et al., Res. Publ. Assoc. Res. Nerv. Ment. Dis., 46 (1968) 399. 